\title{
Comprehensive Evaluation of Housing Safety Appraisal Institutions Based on Fuzzy Analytic Hierarchical Process Method
}

\author{
Yihua $\mathrm{Mao}^{\mathrm{a}}$ and Haiwei Huang ${ }^{\mathrm{b}}$ * \\ Zhejiang University, College of Civil Engineering and Architecture, Hangzhou 310000, China \\ azjumyh@163.com, , ${ }^{*}$, zju_hhw@163.com
}

Keywords: Expert interview, literature review, evaluation model, evaluation index system.

\begin{abstract}
In this paper, evaluation indexes of housing safety appraisal institutions are identified through the expert interviews and literature review. And on this basis, the evaluation index system is established. Then, fuzzy analytic hierarchy process is used to establish the evaluation model, which quantifies the qualitative indexes and achieves the comprehensive evaluation. Finally, this proposed method has been applied to an actual institution, which proves the scientific and feasible of the evaluation model.
\end{abstract}

\section{Introduction}

With the rapid development of urbanization, the number of newly built high-rise buildings has been increasing while the number of houses under construction has also been gradually increasing. Most people tend to pay attention to the management of the construction phase, while ignoring the management of the using phase. The safety assessment as the first step of judging the safety of the building is the most crucial step in the management of the use phase of the building.

For the current situation of the domestic housing safety appraisal market, housing safety appraisal work is generally commissioned by the owners or the real estate department on behalf of the owners entrusted with the housing safety appraisal institutions, housing safety appraisal institutions determine the quality of appraisal. However, the housing safety appraisal market also has the problems of low price competition and non-standard appraisal of practitioners in the appraisal unit. Therefore, it is necessary to make a comprehensive and scientific evaluation of the housing safety appraisal institutions, standardize its appraisal of housing security and establish an honest and trustworthy market environment.

\section{Literature References}

Housing safety appraisal refers to the detection, calculation, analysis and appraisal of the damage degree and using status of house structure according to the basic professional knowledge of mechanics and building structure [1]. Bingjing Mo elaborated on the current situation of the housing safety appraisal and the main contents of the appraisal, and summarized the necessity and application of appraisal work [2]. Dan Yang and Limei Guo established quality management evaluation system and comprehensive evaluation mechanism of the appraisal institutions from analyzing the structure of quality management system [3]. Xue Teng researched the evaluation criteria and model of housing safety appraisal institutions from the point of value chain, organizational structure and other aspects [4]. Yuanhui Liu established an appraisal institutions integrity evaluation system and evaluated the credit data of appraisal institutions, so as to encourage them to strengthen management, improve technical level and professional level [5]. Dongmei Xu and Huidong Yang put forward the idea of credit rating system and established the credit evaluation index system and appraisal method of appraisal institution [6]. Based on the characteristics of the third-party quality appraisal institutions, Wentao Wang and Hengquan Zhang established a second-level index system and used the analytic hierarchy process to evaluate and select the third-party quality appraisal institutions to provide the basis for the owner about choosing a third-party quality appraisal institution [7]. Jiefeng Li and Qiang Xiao analyzed the status of credit management of institutions and put forward suggestions for strengthening credit management based on the actual situation [8]. Ruifeng Guo and Haiying Wan 
conducted a brief analysis on the status of credit construction in construction industry both at home and abroad, and put forward the plan of establishing credit rating system [9].

\section{Constructing Evaluation Index System}

Establishing a comprehensive evaluation index system of housing safety appraisal institutions is divided into two steps of primary and perfect. First of all, through the literature review, this paper analyzes the factors of housing safety appraisal institutions in four aspects: basic conditions, market management activities, appraisal quality, rewards and punishment, and initially establishes the evaluation index system. On this basis, the index is screened to further improve the index system by expert consultation. The final index system includes 14 third-level evaluation indexes. See Table 1 for details.

Table 1. Evaluation Indexes for Housing Safety Appraisal Institution

\begin{tabular}{|c|c|c|}
\hline Target Layer & Criterion Layer & Index Layer \\
\hline \multirow{12}{*}{$\begin{array}{c}\text { Comprehensive Evaluation of Housing } \\
\text { Safety Appraisal Institution P }\end{array}$} & \multirow{5}{*}{ Basic Conditions $\mathrm{P}_{1}$} & Qualification Level $\mathrm{P}_{11}$ \\
\hline & & Technical Staffing $\mathrm{P}_{12}$ \\
\hline & & Instrument and Software $\mathrm{P}_{13}$ \\
\hline & & Institution Performance $\mathrm{P}_{14}$ \\
\hline & & Record Situation $\mathrm{P}_{21}$ \\
\hline & \multirow{2}{*}{ Market Management Activities $\mathrm{P}_{2}$} & Management Behavior $\mathrm{P}_{22}$ \\
\hline & & Market Behavior $\mathrm{P}_{23}$ \\
\hline & \multirow{2}{*}{ Appraisal Quality $\mathrm{P}_{3}$} & Calculation Review $\mathrm{P}_{31}$ \\
\hline & & Appraisal Report $\mathrm{P}_{32}$ \\
\hline & \multirow{3}{*}{ Rewards and Punishment $\mathrm{P}_{4}$} & $\begin{array}{c}\text { Rewards } \mathrm{P}_{41} \\
\text { Social Responsibility } \mathrm{P}_{42}\end{array}$ \\
\hline & & Punishment $\mathrm{P}_{43}$ \\
\hline & & Discipline Information $\mathrm{P}_{44}$ \\
\hline
\end{tabular}

\section{Fuzzy Analytic Hierarchical Process Model}

\subsection{Determine the Factor Set}

The factor set $P$ is the collection of evaluation indexes. Let $P=\left\{P_{1}, P_{2}, \cdots, P_{m}\right\}$. First grade indexes can be classified into second grade indexes. Suppose that $P_{j}$ is reflected by $n$ secondary indexes, then $P_{j}=\left\{P_{i 1}, P_{i 2}, \cdots, P_{i n}\right\}(i=1,2, \cdots, m)$.

\subsection{Determine the Index Weight}

The determination of the weight of the evaluation indexes plays an important role in the objective reflection of the comprehensive abilities of the housing safety appraisal institution. In this paper, analytic hierarchical process is used to determine the index weight, which includes the following steps: constructing the judgment matrix, using sum-product method to solve judgment matrix and consistency check.

\subsection{Determine the Comment Set}

$V=\left(v_{1}, v_{2}, \cdots, v_{N}\right)$ Represents the $N$ evaluation grades of each factor, which specifies the range of evaluation results for a factor. $N$ is an odd number in [3, 7]. In this paper, $N$ is equal to 5 , and each evaluation grade represents the extent to which each index affects the evaluation result.

\subsection{Determine the Membership Matrix}

The key of the fuzzy evaluation method is to analyze the membership degree of each factor in the evaluation factor set for each evaluation grade, so as to get the evaluation result of the single factor.

Assuming that the $i$-th evaluation factor is $P_{i}$, the single factor evaluation result is $X_{i}=\left(x_{i 1}, x_{i 2}, \cdots, x_{i N}\right)$, and $x_{i j}$ is the membership degree of the $i$-th evaluation factor $P_{i}$ to the $j$-th grade. If the $m$ elements are evaluated, finally we will obtain the membership matrix of the $m$ rows and $N$ columns, where $x_{i j}$ can be determined by:

$$
X_{i j}=\frac{d_{i j}}{d}
$$


$d_{i j}$ is the number of people who give the index $P_{i}$ evaluation grade $j$, and $d$ indicates the total number of experts.

\subsection{Fuzzy Synthesis Calculation and Evaluation}

By multiplying the fuzzy weight vector with the membership matrix, we can obtain the membership degree of the evaluation factor as a whole for each evaluation grade. Then we can determine the evaluation level of the evaluation object, and give the evaluation conclusion according to the principle of maximum membership degree, at the same time we can calculate the total score of the project.

\section{Case Analysis}

We take a housing safety appraisal institution as an example and invite 20 experts to evaluate it with the evaluation model.

\subsection{Determine the Index Weight and Test the Consistency.}

Based on the analytic hierarchical process, 20 experts compare the importance of the evaluation factors, and we obtain the judgment matrix: $P-P_{i}, P_{1}-P_{1 j}, P_{2}-P_{2 j}, P_{3}-P_{3 j}, P_{4}-P_{4 j}$. Then calculate the index weight according to the judgment matrix, and test its consistency. The final weight of each element is:

$$
\begin{gathered}
W=(0.0794,0.2568,0.6077,0.0561) \\
W_{1}=(0.0563,0.1425,0.2468,0.0644) \\
W_{2}=(0.6267,0.0936,0.2797) \\
W_{3}=(0.1250,0.8750) \\
W_{4}=(0.0868,0.0564,0.5263,0.3305)
\end{gathered}
$$

$\mathrm{W}$ denotes the index weight in the criterion layer, and $W_{i}$ is the index weight in the index layer.

\subsection{Construct the Comment Set}

After determining the factor set and the index weight, we need to determine the comment set. In this paper, the evaluation grades are divided according to the degree of influence of each index on the institution. The meanings of the specific division method and each evaluation grade are shown in Table 2.

Table 2. Comprehensive Ability Assessment

\begin{tabular}{cc}
\hline Evaluation Grade & Evaluation Comment \\
\hline$(0,1]$ & Very Bad \\
$(1,2]$ & Bad \\
$(2,3]$ & Medium \\
$(3,4]$ & Good \\
$(4,5]$ & Very Good \\
\hline
\end{tabular}

\subsection{Determine the Membership Degree of Each Evaluation Factor}

20 experts evaluate the evaluation grade of each factor according to the above comprehensive performance assessment criteria. And we get membership matrix as follows:

$$
\begin{array}{lllll}
X_{1}= & {\left[\begin{array}{ccccc}
0 & 0.15 & 0.25 & 0.5 & 0.1 \\
0.05 & 0.15 & 0.35 & 0.35 & 0.1 \\
0.2 & 0.2 & 0.3 & 0.3 & 0 \\
0.3 & 0.25 & 0.3 & 0.15 & 0
\end{array}\right]} & X_{2}=\left[\begin{array}{ccccc}
0 & 0.2 & 0.2 & 0.4 & 0.2 \\
0.2 & 0.3 & 0.25 & 0.2 & 0.05 \\
0.15 & 0.35 & 0.25 & 0.15 & 0.1
\end{array}\right] \\
X_{3}=\left[\begin{array}{ccccc}
0.3 & 0.15 & 0.35 & 0.2 & 0 \\
0 & 0.2 & 0.25 & 0.2 & 0.35
\end{array}\right] & X_{3}=\left[\begin{array}{ccccc}
0.25 & 0.2 & 0.25 & 0.2 & 0.1 \\
0.15 & 0.3 & 0.35 & 0.15 & 0.05 \\
0 & 0.15 & 0.2 & 0.4 & 0.25 \\
0.05 & 0.15 & 0.35 & 0.2 & 0.25
\end{array}\right]
\end{array}
$$

\subsection{Fuzzy Evaluation}

Multiply the evaluation matrix of index (second index) and the weight of the evaluation index to obtained evaluation matrix of evaluation index (secondary index) in criterion layer, where: 


$$
\begin{gathered}
B_{i}=W_{i} X_{i} \\
B_{1}=(0.0758,0.1688,0.2798,0.4067,0.0689) \\
B_{2}=(0.0607,0.2513,0.2187,0.3113,0.1580) \\
B_{3}=(0.0375,0.1938,0.2625,0.2000,0.3062) \\
B_{4}=(0.0467,0.1628,0.2624,0.3024,0.2257)
\end{gathered}
$$

In the same way, multiply the index weight of the criterion layer and evaluation weight to obtain the evaluation matrix of the target layer:

$$
B=W X=(0.0470,0.2048,0.2526,0.2508,0.2448)
$$

In order to get the performance score of the institution, we take the median of each evaluation grade to get the vector $K=(0.5,1.5,2.5,3.5,4.5)$. So the comprehensive score is:

$$
S=B K^{T}=2.9415
$$

We can find that the institution has the highest membership degree for the evaluation grade $(2,3]$ from B. The overall score of 2.9415 also shows that the evaluation of the institution is medium.

\section{Conclusion}

In this paper, we construct the evaluation index system according to the characteristics of housing safety appraisal institutions, and establish the evaluation model by using fuzzy analytic hierarchical process method to evaluate the comprehensive ability. Through the ability assessment, we can understand the main factors that determine the ability of housing safety appraisal institutions, so as to broaden the thinking of the research on housing safety appraisal institution, and also provide the housing owners with effective suggestions about how to choose a better housing safety appraisal institution.

\section{References}

[1]. J.M Chen: Housing Safety Appraisal Practice (China Architecture \& Building Press, China 2014), pp. 121-127.

[2]. B.J. Mo: Sichuan Building Materials, Vol. 43 (2017) No.5, pp. 37-39.

[3]. D. Yang and L.M. Guo: Construction Quality, Vol. 34 (2016) No.12, pp. 66-68.

[4]. X. Teng: Construction Engineering Quality Inspection Company Core Competitiveness Evaluation Research (MS., Dalian University of Technology, China 2016), pp. 34-38.

[5]. Y.H. Liu: Guangzhou Architecture, Vol. 63 (2015) No.3, pp. 45-47.

[6]. D.M. Xu and H.D Yang: Shanxi Architecture, Vol. 41 (2015) No.29, pp. 239-241.

[7]. W.T. Wang and H.Q. Zhang: Project Management Technology, Vol. 12 (2014) No.4, pp. 43-46.

[8]. J.F. Li and Q. Xiao: Construction Quality, Vol. 32 (2014) No.2, pp. 291-294.

[9]. R.F. Guo and H.Y. Wang: Anhui Architecture, Vol. 33 (2014) No.3, pp. 189-193. 